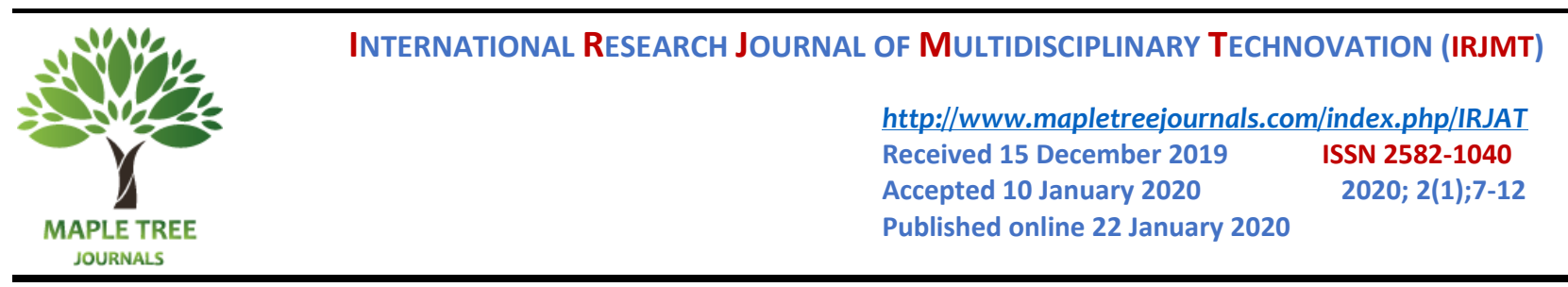

\title{
Automatic Sorting Machine
}

\author{
Aashika Prasad 1, *, M. Gowtham ${ }^{1}$, S. Mohanraman ${ }^{1}$, M. Suresh ${ }^{1}$ \\ ${ }^{1}$ Department of Robotics and Automation, PSG College of Technology, Coimbatore, Tamil Nadu, India. \\ *Corresponding author E-Mail ID: aashikaaprasad@gmail.com Mobile: +91-8124199433 \\ DOI: https://doi.org/10.34256/irjmt2102
}

\begin{abstract}
Businesses around the world automate their outbound profiling and sortation process for an increased productivity. Sorting plays a key role in any industrial automation, particularly in the field of packaging industries. This paper aims at providing significant low-cost automation in packaging industries that calls for sorting and how the implication of pneumatics can yield a desirable solution. The prototype of the automatic sorting machine allows a systematic sorting of the carton boxes corresponding to their sizes using a pneumatic system, which is achieved by using pneumatic cylinders and direction control valves. The sorted carton boxes are shoved from the main line conveyor to the sub-line conveyor using the pneumatic cylinder, which is then collected and dispatched to the next division for the packaging purposes. Using this approach, the packaging industries can attain the maximum benefits thereby enabling a surge in their production.
\end{abstract}

Keywords: Automatic sorting machine, pneumatic system, packaging industries, low-cost automation.

\section{INTRODUCTION}

Over the last two decades, it is clearly evident that automation is being implemented in all the labour-intensive industries. Recent trends in automation are very well discerned in the packing industries. Packaging industries were born right after the surging need for packaged products to be transported and stored with no damage. During the initial stages of the evolution of the packaging industries, packaging was carried out using wooden boxes, plastic and paper bags. As the trends in the packaging industries keep increasing, there is a necessity for the automation of sorting the cartons according to their size. This can be achieved by using the pneumatic system, which saves labour cost and time, thereby increasing the accuracy and overall efficiency leading to increased productivity and profits.

\section{RELATED WORKS}

The concept of automatic sorting arose due to the difficulties faced by the packaging industries. The idea of sorting has existed for quite a while after there have been advancements in the technology.

Shen and Hassan [1] states that an approach for continuous recognition and the sorting of objects into their respective and desired location can be implemented as an image of colour processing that can attract an enormous attention leading to a possible widening scope of application in a different field in a modern technology. A colour-sorting robot is designed and developed using an Arduino Uno microcontroller, SG90 Tower Pro Servo Motor, TCS3200D colour sensor and several other electronic components. The system has the potential to sort the 
objects according to their colours into their relevant colour station in a less time. A distinct code for this system is developed.

According to Yunardi et al. [2], a 3D volume of the packed box is well quantified from the $2 \mathrm{D}$ images using the techniques of image processing. The 2D image consists of two images captured on the camera with a horizontal view and a vertical view. With the parameters, that is, the length, width and the height, a multiplication program is used to obtain the result of the volume. Consequently, contour based object detection can be appertained to the automatic sorting system to measure the volume of an object in a computer-based vision.

According to Babita [3], sensors are embedded at several places that detects and senses the materials of various sizes that gets sorted at different stations based on their sizes. For sensing, the material used is an infrared sensor that is so sensitive. All the process is controlled and handled by a PLC.

Kulkarni et al. [4] has implemented the sorting of the boxes using a barcode, which is decoded by raspberry pi. This raspberry pi sends a signal to the motor driver to start and stop the motor accordingly. Proximity switches are implanted on every cylinder to sense the box position, which again sends back the signal to raspberry pi. Relay is used as an interface for the motor driver and motor. The relay used is of solid relay type. Raspberry pi uses cameras of five megapixels to capture the image, which will then be decoded.

The sorting process is controlled and handled by Arduino Microcontroller. Automating every sector of industry is an important step towards increasing efficiency and reducing human related errors, here we try to automate the sorting process by using Controller. The program fed in the Arduino controls the entire process. [5]

\section{PROTOTYPE DESIGN}

The prototype of the pneumatic circuit is designed using two double acting pneumatic cylinders, 3/2 roller-operated spring-return direction control valves, 5/2 pilot-operated springreturn direction control valves, an FRL unit and a compressor unit. The number of the double acting pneumatic cylinders and the direction control valves will vary according to the corresponding number of sizes of carton boxes to be sorted for a required application.

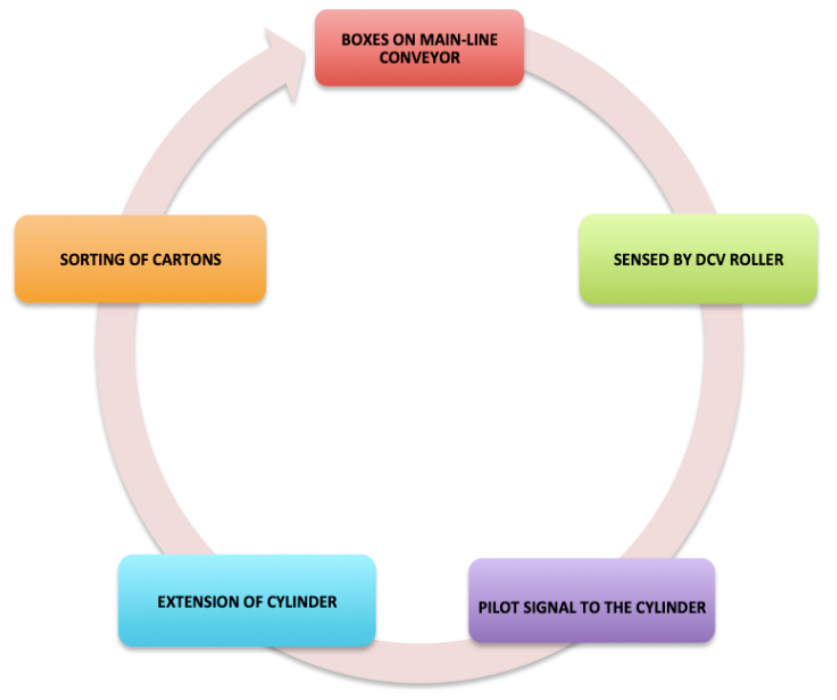

Figure 1. Block diagram of the prototype 


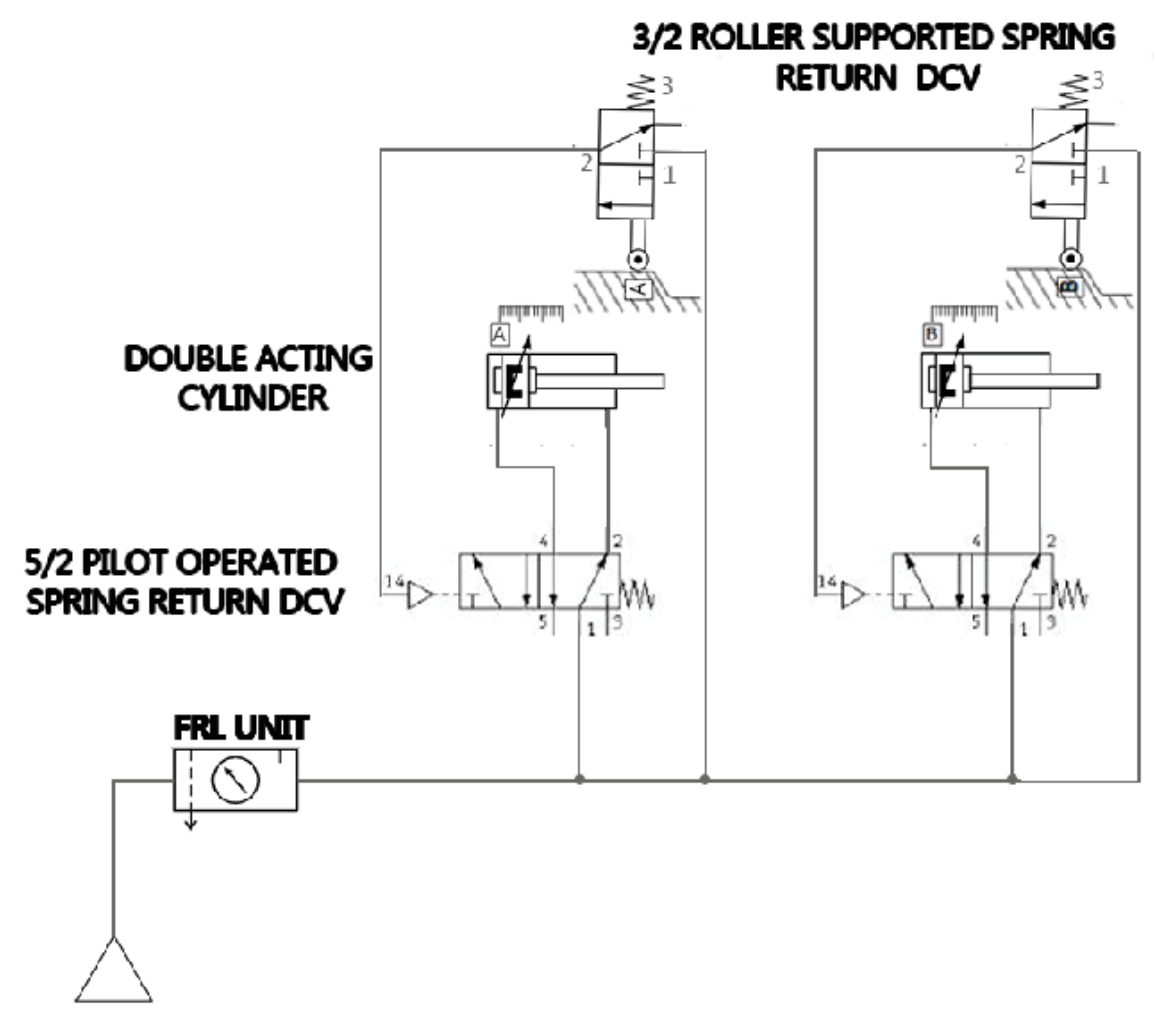

Figure 2. Pneumatic circuit for the prototype

\section{WORKING OF THE PROTOTYPE}

The air compressor that stores the compressed air in it releases the compressed air to the system that is under control through the FRL unit. The cylinders are fixed at descending order of heights in proportion to the sizes of the cartons, that is, the second roller operated DCV is kept at a lower height than the first and so on, so as to not encounter any mismatch in sorting.

As the carton boxes travelling in the main-line conveyor nears the cylinder, the roller of the respective $3 / 2$ roller-operated spring-return direction control valve senses the touch of the carton, thereby the position of the valves changes forcing the compressed air into the port of one end of the cylinder.

This results in pushing the piston forward resulting in extension of the cylinder, thereby sorting the carton into the respective sub-line conveyor belt. The alternating pressure moves the boxes to the specified distance and then the piston of the cylinder retracts back after this process for the next forward stroke. This process is followed for the cartons of all sizes.

In addition, a soft spring is attached on one side of the main-line conveyor, which allows a huge flexibility for the cartons to adjust itself according to the sizes by extension and contraction of the spring. According to the industrial needs, the range of the spring constants can be chosen such that it aids the boxes from smallest to the largest sizes to pass through the spring channel. 


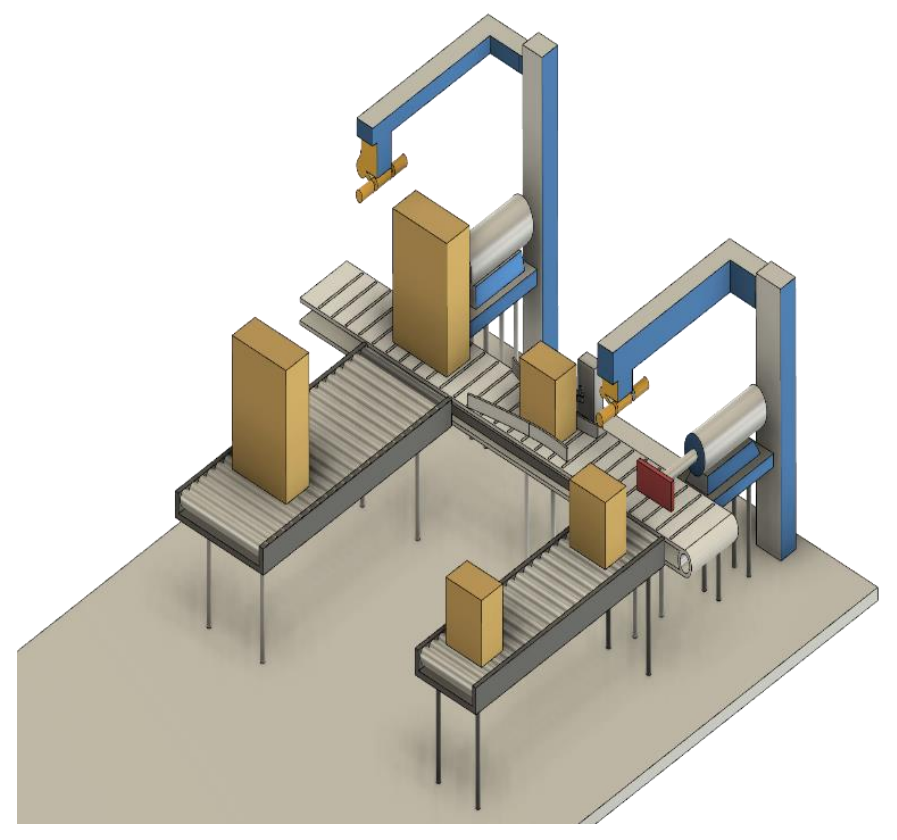

Figure 3. Solidworks model for the prototype

\section{DESIGN PARAMETERS}

Design parameters are the key and fundamental considerations to be made for any automation. The parameters to be considered for this prototype are the type of cylinders, the kind of direction control valves, the pressure range, mounting and control option and most importantly the bore and stroke length of the cylinder.

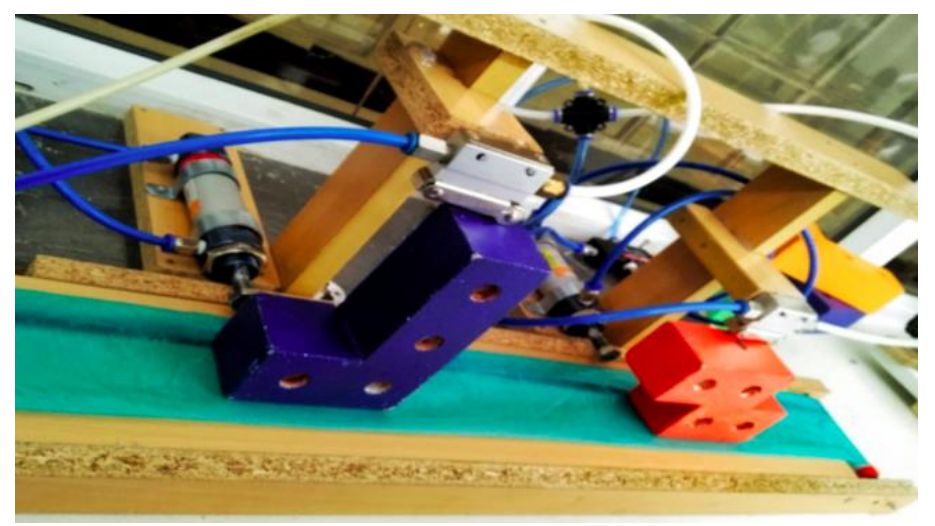

Figure 4. Designed prototype model

\section{RESULTS}

The experimental setup was carried out to sort the carton boxes according to their sizes. The sizes considered for the experiment are cartons of 5 centimeters and 10 centimeters. The roller of the first $3 / 2$ roller-operated DCV is named as A and the roller of the second is named as B. The roller A and B are mounted at a height of 10 centimeters and 5 centimeters respectively. The findings of this experiment are explained and illustrated as follows.

Case 1: Sorting of the carton boxes of 10 centimeters 
As the carton box of 10 centimeters move along the main-line conveyor, the roller $\mathrm{A}$ senses the carton and sorts the box to its respective sub-line conveyor. The roller B has no action on this carton box.

\section{Case 2: Sorting of the carton boxes of 5 centimeters}

As the carton box of 5 centimeters move along the main-line conveyor, the roller A does not sense the carton and has no action on the box and travels to the roller B where the carton is sensed and the box is sorted to its respective sub-line conveyor.

Case 3: Sorting of the carton boxes neither of 5 centimeters or 10 centimeters

The carton boxes that are neither 5 centimeters nor 10 centimeters does not get sensed by the roller A as well as roller B and is not sorted. For this, an additional cylinder has to be set up to sort the cartons of the required sizes.

In addition to this, a counter can be added to the circuit if required, so as to count the number of boxes that is sorted into each sub-line conveyor.

Table 1. Experimental results

\begin{tabular}{|l|l|l|l|l|}
\hline CASE & CARTON SIZE & ROLLER A & ROLLER B & STATUS \\
\hline 1 & $10 \mathrm{~cm}$ & Sensed & No action & Sorted \\
\hline 2 & $5 \mathrm{~cm}$ & No action & Sensed & Sorted \\
\hline 3 & $8 \mathrm{~cm}$ & No action & No action & Not sorted \\
\hline 4 & $15 \mathrm{~cm}$ & No action & No action & Not sorted \\
\hline
\end{tabular}

\section{CONCLUSION}

The Automatic Sorting Machine works effectively and makes sorting process easy, more precise and reliable and is more advantageous than the conventional methods reducing manual efforts, errors and being much efficient. The proposed prototype allows achieving an economical and a low-cost automation. The sorting action can be made flexible according to the industrial needs. In case of any breakdown, the system can be easily restored and commissioned upon diagnosis.

\section{REFERENCES}

[1] Lim Jie Shen \& Irda Hassan, Design and Development of Colour Sorting Robot, Journal of Engineering Science and Technology EURECA 2014, (2015), 71-81. http://jestec.taylors.edu.my/eureca2014(1)_1_2015/eureca_14(1)_71_81.pdf

[2] Riky Tri Yunardi, Winarno and Pujiyanto, Contour-based object detection in Automatic Sorting System for a parcel boxes, International Conference on Advanced Mechatronics, Intelligent Manufacture, and Industrial Automation (ICAMIMIA), (2015) 38-41, DOI: https://doi.org/10.1109/ICAMIMIA.2015.7507998

[3] Babita Nanda, Automatic Sorting Machine using Delta PLC, International Journal of Innovative Research in Advanced Engineering, 1(7) (2014) 235-242. 
[4] Moe Win Khaing, Aye Mya Win and Daw Thida Aye, Automatic Sorting Machine, International Journal of Science and Engineering Applications, 7(8), (2018) 138-142. DOI: https://doi.org/10.7753/IJSEA0708.1002

[5] Shreeya Vasant Kulkarni, Swati Rajendra Bhosale and Priyanka Pramod Bandewar, Automatic Box Sorting Machine, International Journal for Scientific Research \& Development, 4(4) (2016), 57-58.

[6] Yoshito Nagaoka, Tomo Miyazaki, Yoshihiro Sugaya and Shinichiro Omachi, Automaic Mackeral Sorting Machine Using Global and Local Features, IEEE Access Volume:7, (2019) 63767-63777. DOI: https://doi.org/10.1109/ACCESS.2019.2917554

[7] Yunhan Lin, Haotian Zhou, Mingy Chen and Huasong Min, Automatic sorting system for industrial robot with 3D visual perception and natural language interaction, Measurement and Control,52(1-2),100-115. https://doi.org/10.1177/0020294018819552

[8] Masao Sugiyama, Automatic Sheet Sorting Machine, Japan Tappi Journal, 52(1) (1998), 8893. DOI: https://doi.org/10.2524/jtappij.52.88

[9] Xian Ke Ren, Dong Ming Li, Hang Zhang, Yun Xiang Cui, Automatic Sorting Machine for Cylinder Length, Advanced Materials Research, (706-708) (2013), 1222-1225. DOI: https://doi.org/10.4028/www.scientific.net/AMR.706-708.1222

[10] Li Peilin, Yang Zhen, Zhou Wenlong and Lu Hong, An automatic sorting system for sorting metal cylindrical workpiece based on machine vision and PLC technology, 2nd International Conference on Robotics and Automation Engineering (ICRAE), Shanghai, (2017), 446-450. DOI: https://doi.org/10.1109/ICRAE.2017.8291427

\section{Acknowledgement Nil}

\section{Funding}

This study was not funded by any grant

\section{Conflict of interest}

None of the authors have any conflicts of interest to declare.

\section{About The License}

The text of this article is licensed under a Creative Commons Attribution 4.0 International License

\section{Cite this Article}

Aashika Prasad, M. Gowtham, S. Mohanraman, M. Suresh, Automatic Sorting Machine, International Research Journal of Multidisciplinary Technovation, Vol 2, Iss 1 (2020) 7-12.

DOI: https://doi.org/10.34256/irimt2102 